\title{
Management of patients with carotid stenosis
}

\author{
A. De Fabritiis, E. Conti, S. Coccheri \\ Division of Angiology - S.Orsola-Malpighi Hospital, University of Bologna, Italy
}

\section{Key Words}

Carotid atherosclerosis, echo-Doppler, angiography, carotid endarterectomy, antiplatelet drugs.

\begin{abstract}
Carotid atherosclerosis is one of the main risk factors for ischemic stroke. The annual risk of ipsilateral stroke for asymptomatic, albeit severe stenoses is as low as 1 to $2 \%$, but increases to $13 \%$ in patients with recent ischemic symptoms. However the risk decreases after the first 2-3 years from the symptomatic episode, dropping to $3 \%$.

Echo-color Doppler ultrasonography is the screening method of choice, being highly accurate, noninvasive and low-cost. Carotid angiography still represents the gold standard, however, less invasive techniques as RM angiography and Angio-CT are becoming increasingly common.

Based on NASCET, ECST and ACAS results, carotid endarterectomy (CE) is strongly recommended for severe symptomatic stenoses, while for the moderate symptomatic and the severe asymptomatic ones the benefit in terms of stroke risk reduction is modest and surgery should be restricted to selected cases in surgical centers of high experience. For severe asympto-
\end{abstract}

matic stenoses NNT is too high to recommend indiscriminate surgery; we are waiting for the results of ACSRS trial, designed to identify a subset of patients at risk of ipsilateral stroke greater than $4 \% / y$, that may be considered for $\mathrm{CE}$, while patients at low risk will be spared from unnecessary operation.

Apart from surgery, in all patients with carotid atherosclerosis correction of cardiovascular risk factors is mandatory. Antiplatelet therapy (ASA alone or with dypiridamole, ticlopidine) is effective in secondary prophylaxis of athero-thrombotic stroke; its use in asymptomatic carotid stenoses can be recommended, even if more because of a plausible rationale than of clinical trial-based evidences.

Copyright @ 2002 S. Karger AG. Basel

\section{Introduction}

In industrialized countries strokes are the third major cause of death and the main cause of invalidity. In $15-20 \%$ of cases strokes are caused by atheromasic lesions at the carotid bifurcation, through either hemodynamic (low-flow infarct) or more usually - atheroembolic mechanism. In the recently published Italian guidelines on the prevention of stroke - the socalled SPREAD (Stroke Prevention and Educational Awareness Diffusion) (1) - a good deal of space is given over to carotid stenosis on account of its important - and modifiable

\section{KARGER \\ Fax +41613061234 \\ E-Mail karger@karger.ch}

www.karger.com (c) 2002 S. Karger AG. Basel

1424-8832/02/0326-0381\$18.50/0

Accessibile online at: www.karger.com/journals/pht
Prof. Sergio Coccheri

Div. of Angiology

Hospital S. Orsola-Malpighi

Via Massarenti, 9

40138 Bologna

E-Mail: coccheri@med.unibo.it 
- role as risk factor for stroke.

The factors at play in the formation/progression of carotid plaque, which are linked to embolization or reduction of cerebral blood flow, are still today not well known, despite numerous studies on the issue. From anatomical-pathological studies we know that in most cases also repair mechanisms that remodel the carotid lesion exist though very little is known about their genesis.

\section{Carotid stenosis and risk of stroke}

The frequency of carotid artery disease in the general population steadily increases with age (from $0.5 \%$ under 60 years to $10 \%$ above 80 ). Various studies have tried to trace the natural history of carotid plaque, its rate of progression and the risk of stroke. A lot of the data have been collected retrospectively and very few prospective studies have been large enough to provide a reliable estimate of the risk of stroke according to degree of carotid stenosis. The ACAS study (Asymptomatic Carotid Atherosclerosis Study) (2) evaluating the benefit of endarterectomy for severe asymptomatic stenoses showed that patients treated with medical therapy only run an $11 \%$ risk of homolateral stroke in 5 years. The data on the contralateral asymptomatic carotids of the 2295 patients included in the ECST study (European Carotid Surgery Trial) (4) had the advantage over the ACAS study of covering a wide range of stenoses: the risk of stroke from a mild stenosis $(<30 \%)$ is no different to that from a moderate one $(30-69 \%)$, equal to $1.8 \%$ and $2.1 \%$ respectively at 3 years. The risk increases to $5.7 \%$ in the $70-99 \%$ class. In the two studies cited the yearly risk of stroke in patients with severe carotid stenosis is virtually the same $(2.2 \%$ and $1.9 \%$ respectively).

The data collected from population studies differ. In the Cardiovascular Health Study (CHS) (3) that examined 5000 subjects aged over 65 years, $57 \%$ of whom were women, the percentage of severe stenoses $(>70 \%)$ was $0.5 \%$ with an annual risk of homolateral stroke equal to $1 \%$. Though older than the patients in the ACAS and ECST studies, the subjects enrolled in this epidemiological study presented less cardiovascular risk factors and a different gender mix with more women. Adjusting the ACAS data for gender, a lower risk of stroke in women is confirmed (1.7\%/y vs $2.4 \% / y$ in men).

In view of the fact that the overwhelming majority of patients with severe carotid stenosis remain aymptomatic, other factors besides the degree of stenosis must be important in determining whether the lesion will become clinically symptomatic. Plaques that have a predisposition for breaking or cracking ("vulnerable" plaque) may be at greater risk of embolization or occlusion of the lumen, and hence of ischemic episodes. In the North American Symptomatic Carotid Endarterectomy Trial (NASCET) (5) angiography identified ulcered lesions in one third of severe stenoses. After two years a non fatal stroke or vascular death occurred in $30 \%$ of the patients with ulcers and in $17 \%$ of those without ulcers, the gap growing steadily as the stenosis became higher, reaching $73.2 \%$ and $21.3 \%$ for stenoses of $95 \%$. In a recent series of 1252 symptomatic and asymptomatic patients submitted to endarterectomy (9) intra-plaque hemorrhaging was found more frequently in $>90 \%$ stenoses $(68 \%$ versus $32 \%$ ) but was not associated with the presence of symptoms, unlike in the case of macroscopic diagnosis of ulcerated plaque (78\% versus $60 \%$ ).

According to a recent review of 11 observational studies published between 1960 and 1995 (7) symptomatic carotid occlusion is associated with an annual risk of stroke equal to $6 \%$ (homolateral 2\%). The biggest of these trials, the EC/IC Bypass Study Group published in 1985 (8), showed that extraintracranial bypass was no more efficacious than only medical therapy in reducing the risk of stroke in patients with symptomatic carotid occlusion.

\section{Clinical presentation of carotid stenosis}

The definition of asymptomatic stenosis is not clear-cut or straightforward as neither is the corresponding risk of stroke. At one extreme we find subjects with few or none of the known cardiovascular risk factors who present no neurological signs and symptoms or atherosclerosis in other areas; the brain scan is negative and the stenosis is detected via an epidemiological survey or by the presence of a cervical bruit. At the other extreme we find the polyvasculopathic subject who presents silent ischemic lesions of the homolateral hemisphere, and/or contralateral previous symptoms or carotid endarterectomy. Since the risk of stroke increases steadily from one extreme to the other, the heterogeneousness of the definition of "asymptomatic stenosis" needs to be kept in mind when trying to apply trial data to everyday clinical practice. Furthermore, the prevalence of disease one might expect in a particular population has a major role in determining the number of false and true positives in any screening test, regardless of diagnostic accuracy.

A carotid stenosis is said to be symptomatic when associated with homolateral retinal and/or hemispheric deficits, even in the absence of lesions at CT scan. Excluded are aspecific symptoms such as syncope or occasional dizziness even if, on account of their frequency in subjects of all ages, these are disorders for which echo-Doppler is most often requested. Depending on the duration of the symptoms, the ischemic events are divided into transient ischemic attacks (TIA) and stroke.

The time limit for defining TIA is 24 hours even if in most cases the symptoms disappear within an hour. In the Cooperative Study of Transient Ischemic Attacks (6) it was observed that if the symptom was present for more than one 
hour only in $14 \%$ of cases would it resolve within 24 hours. Carotid TIA are usually stereotyped in that the same vascular area is involved. An isolated TIA occurring more than two weeks before observation doesn't require hospitalization, providing of course the facilities are in place for examining the patient quickly. Such an occurrence is not rare. In the ACAS study, even though patients were instructed to report immediately the onset of any neurological symptom, reporting within 3 days occurred in less than $40 \%$. If the time between symptoms is less than 2 weeks, the decision on hospitalization depends on the seriousness of the symptom or - if multiple by the increase in duration and severity ("crescendo" TIA).

Minor-stroke is characterized by an ischemic neurological deficit lasting over 24 hours, with good recovery (Rankin scale 1-2). A different classification uses a time interval of 7 days to distinguish minor and major strokes, with major strokes then being subdivided into non-incapacitating and incapacitating (Rankin scale 3-4-5), at 3 o 6 months from onset of the symptoms.

\section{Diagnosis of carotid stenosis}

In clinical practice carotid artery plaque is normally studied by angiography and ultrasound. Using bi-planar projections, angiography still represents the gold standard for evaluating the extent of stenosis of the vessel lumen caused by plaque. There are two different methods, used in the two most important trials on the efficacy of carotid endartectomy in reducing the risk of stroke in patients with symptomatic stenosis (9): the "E" (European) and the "N" (North American) methods. The degree of stenosis is measured as the ratio between minimum residual lumen and the vessel diameter, at the level of stenosis (E) or distal to stenosis $(\mathrm{N})$. Because the most frequent site of pathology is the bulb, the presumed diameter of the vessel at the level of stenosis will be larger than the distal segment in the most of the cases: a stenosis of $70 \%$ using method "E" corresponds to a stenosis of just under $50 \%$ with method "N" while a stenosis of $70 \%$ using method "N" corresponds to a stenosis of just over $80 \%$ with method "E" (10). Since angiography is not risk-free (1.2\% of strokes or deaths in the ACAS study) patients should be screened by non-invasive methods.

Using echo-Doppler the degree of stenosis is derived from blood flow velocity data. The single most used parameter is peak systolic velocity (PSV) in the point of maximum stenosis, which can identify severe stenosis with a diagnostic accuracy of over $90 \%$ (93\% in the ACAS trial). The angiographic method of reference used to define the degree of stenosis ( $E$ or N) must be specified. Different threshold values have been proposed in the literature but it has recently been recognized that their validation has to be laboratory-specific. Generally speaking, if the decision to perform surgery is taken solely on the basis of ultrasound data, the cut-off value must be such as to reduce false positives to a minimum; if, on the other hand, the next step is angiography a lower value can be chosen which reduces the false negatives. The diagnosis of complete occlusion in a symptomatic patient should always be confirmed by angiography because of the limitations of ultrasound examination to detect very low blood flow (pseudo-occlusion).

Transcranial Doppler complements echo-Doppler in evaluating carotid steno-occlusion to the extent that it provides information on both hemodynamic factors (reduction of middle cerebral artery flow velocity and pulsatility; revascularization via communicating arteries) and on embolic potential (monitoring of microemboli).

B-mode ultrasonography can identify the morpho-structural characteristics of carotid plaque. In the literature diagnostic accuracy of endoluminal ulceration varies greatly (from 39\% to $97 \%$ ), not helped by the lack of standardization. As regards lesion ecogenicity, we can distinguish between hypoecogenic and hyperecogenic plaque, the latter corresponding, from an anatomical-pathological point of view, to fibrocalcific lesions (often asymptomatic in the elderly). Hypo- or an-ecogenic areas within dishomogeneous plaque on the other hand are related to lipidic formations or blood lacunae (intra-plaque hemorrhage), both markers of plaque instability. With a view to overcoming the subjectivity of interpretation, computerized analysis of ecogenicity has been suggested.

Other non-invasive techniques have recently been introduced to evaluate carotid stenosis. Magnetic resonance angiography (MRA) is a technique that is less operator dependent than eco-Doppler with the added advantage that it can offer accurate pictures of intracranial vessels. Like ecoDoppler it has problems in differentiating sub-occlusive stenoses from complete occlusions. However, eco-Doppler and MRA used together have a diagnostic accuracy close to $100 \%$ when the results of the two techniques are concordant, leaving traditional angiography for use only when results are discordant. Angio-CT is also finding its way into clinical practice and in many centers is already replacing traditional angiography for pre-operative evaluation (1).

\section{Cardiovascular risk factors and carotid stenosis}

Arterial hypertension is one of the main risk factors for stroke and the benefit of anti-hypertensive therapy is greater in patients with previous cerebrovascular episodes; generally speaking a reduction in diastolic pressure equal to $5-6 \mathrm{mmHg}$ and of systolic pressure equal to $10-12 \mathrm{mmHg}$ equates to $33-$ $50 \%$ fall in the number of cerebrovascular events.

The aforementioned Cardiovascular Health Study assessed the relationship between carotid atherosclerosis and cardiovascular risk factors, adjusted for age and sex. The size of the stenosis was closely tied to systolic pressure, smoking, dia- 
betes and HDL-cholesterol (inverse relationship). Other important epidemiological studies (Kuopio Ischemic Heart Disease Risk Factor Study and Atherosclerosis Risk in Communities - ARIC study) (13) have pointed up the effects of systolic hypertension and smoking in accelerating the progression of carotid atherosclerosis.

\section{Long-term pharmacological therapy}

An analysis of the Antiplatelet Trialists' Collaboration regarding 100,000 patients in 145 trials up to 1990 showed that antiplatelet drugs reduce the risk of non-fatal stroke by $23 \%$ in patients with previous TIA or minor stroke. In clinical practice it is widespread to treat asymptomatic carotid stenoses too, even if this indication has not been the subject of any controlled study. It should be pointed out however that a study on asymptomatic stenosis in which the surgical group did not receive antiaggregants was suspended on account of an unacceptably high incidence in the group of myocardial infarction. In the Ticlopidine Aspirin Stroke Study (TASS) (12), ticlopidine proved to be only slightly better than aspirin in the secondary prevention of stroke while the possible side effects (myelodepression) and the greater cost have made it a secondchoice drug, reserved for patients with intolerance to aspirin or who get no benefit from aspirin in the prevention of stroke recurrence. Clopidogrel, a derivative of ticlopidine but without its hematological toxicity, proved no more efficacious than aspirin in the subgroup of patients recruited after minor stroke (CAPRIE study, Clopidogrel versus Aspirin in Patients at Risk of Ischemic Events) (11) .

\section{Surgical therapy}

SYMPTOMATIC CAROTID STENOSIS - Two randomized and prospective multi-center trials, the European ECST (4) and the North American NASCET (5), showed incontrovertibly that in patients with recent TIA or minor stroke caused by a carotid stenosis $>70 \%$ endarterectomy significantly reduces the risk of stroke.

In the ECST study 3024 patients were randomized with a mean follow-up of 6 years. The incidence of major stroke or death in the first 30 days after surgery was $7 \%$ (3.5\% of which were non incapacitating strokes). In the controls the risk of stroke was correlated with the severity of the stenosis, but only for the first 2-3 years from initial event. In this period of time and for stenoses $>80 \%$ the absolute reduction in the risk of major strokes or death in the surgery group was $11.6 \%(14.9 \%$ versus $26.5 \%$ ), corresponding to an NNT (number needed to treat) of 9 patients to save one death or one major stroke in 3 years. The perioperative risk was greater in women who would probably benefit if surgery was limited to stenoses $>90 \%$. As the risk of stroke rapidly decreases with the passing of time from the event it is obvious that operating as quickly as possible offers maximum advantage.

In the NASCET study the rate of perioperative major strokes or deaths among patients with stenoses $>70 \%$ was $2.1 \%$. At the end of the follow up period (8 years) an incapacitating homolateral stroke occurred in a further $4.6 \%$ of patients submitted to endarterectomy. The absolute reduction in the risk of homolateral stroke at 2 years was $17 \%$ (9\% in surgery patients, $26 \%$ in controls), corresponding to an NNT of 6 patients to prevent an adverse event. For stenoses in the $50-69 \%$ range the risk of homolateral stroke at 5 years was $15.7 \%$ in surgery patients and $22.2 \%$ in controls $(\mathrm{p}=0.045)$, corresponding to an NNT of 15 patients to prevent one stroke in 5 years. This benefit does not present in women because of the greater perioperative risk they run and the lesser risk they have using just medical therapy. For both moderate and severe stenoses the risk of stroke in the non-surgery group gradually fell to about $3 \%$ per year within 2-3 years. The NASCET study analysed different sub-groups of patients which, though limited because of the low number of subjects involved, nonetheless provided interesting data regarding therapeutic decisions to be taken in specific cases. For example it was suggested that while patients with preocclusive stenosis could benefit from surgery, an emergency operation was not necessary; that the risk-benefit ratio favors surgery in moderate stenoses associated with intracranial lesions and in severe stenoses associated with contralateral occlusion.

ASYMPTOMATIC CAROTID STENOSIS - A recent review examined 5 controlled randomized trials, whose results were published between 1984 and 1995 (7). The trials involved a total of 1215 surgically treated patients and 1225 controls in medical arm, with $74 \%$ of subjects being males and follow up varying between 2 and 5 years. In all studies the size of the stenosis (that varied between $50 \%$ and $99 \%$ ) was confirmed angiographically before surgery. The review pointed to an absolute reduction of $2 \%$ in the risk of homolateral stroke over 3 years (4.4\% versus $6.4 \%$ in controls). This translates into an NNT of 50 to prevent 1 episode in three years. Given that the benefit of surgery is modest, the authors of the review concluded that unless sub-groups of subjects at high risk are reliably identified medical treatment is an acceptable alternative for many patients with asymptomatic carotid stenosis.

The aim of the ongoing multi-center trial ACSRS (Asymptomatic Carotid Stenosis and Risk of Stroke) is to identify a subgroup of patients with an annual risk of stroke $>4 \%$. In this study the patients - all treated with medical therapy - are followed for 5 years with half-yearly clinical and standardized echo-color Doppler examination to evaluate the role of "local" risk factors of stroke (14).

CAROTID ANGIOPLASTY AND STENT - Carotid transluminal percutaneous angioplasty is an alternative to endarterectomy but to date there are no conclusive data from 
controlled and randomized studies that compare the two techniques. This procedure is currently indicated in selected cases such as restenosis and stenoses located both proximally (common carotid) and distally to the carotid bifurcation.

\section{Conclusions}

In all patients with TIA or minor stroke an echo-colordoppler of the supra-aortic trunks should be carried out. If severe stenosis is diagnosed the best treatment is endarterectomy. For moderate stenoses as well as asymptomatic severe stenoses surgery is only modestly beneficial and the therapeutic decision must take full account of the operative risk of each patient. Prescribing an antiplatelet agent may be a valid alternative provided the patients are given careful clinical-instrumental follow-up: the key factor in the non-surgical approach is that the patient is instructed to report immediately the onset of any neurological symptoms.

Correction of cardiovascular risk factors by means of behaviour modifications and drugs is a major goal. Antiplatelet agents reduce the overall risk of cardiovascular events in patients with atherosclerotic vascular disease, whatever its location. The drug of choice is low-dose aspirin (100$300 \mathrm{mg}$ ). In cases of intolerance to or inefficacy of aspirin in preventing recurrence of stroke, ticlopidine or clopidogrel should be used. For other drugs the research data are not conclusive.

\section{References}

1. SPREAD - Stroke Prevention And Educational Awareness Diffusion. Ictus cerebrale: linee guida italiane. Stesura del 20 febbraio 2001. Pubblicazioni Health srl, Milano, 2001.

2. Executive Committee for the Asymptomatic Carotid Atherosclerosis Study: Endarterectomy for asymptomatic carotid artery stenosis. JAMA 1995, 273:1421-1428.

3. Longstreth WT, Shemanski L, Lefkowitz D, O'Leary DH, Polak JF, Wolfson SK for the Cardiovascular Health Study Collaborative Research Group: Asymptomatic Internal Carotid Artery Stenosis Defined by Ultrasound and the Risk of Subsequent Stroke in the Elderly. Stroke 1998; 29:2371-2376.

4. European Carotid Surgery Trialist' Collaborative Group: Randomised trial of endarterectomy for recently symptomatic carotid stenosis: final results of the MRC European Carotid Surgery Trial (ECST). Lancet 1998, 351:1379-1387.

5. Barnett HJM, Taylor DW, Eliasziw M, Fox AJ, Ferguson GG, Haynes RB, Rankin RN, Clagett GP, Hachinski VC, Sackett DL, Thorpe KE, Meldrum MM and HE for the North American Symptomatic Carotid
Endarterectomy Trial Collaborators: Benefit of carotid endarterectomy in patients with symptomatic moderate or severe stenosis. N Engl J Med 1998; 339: 1415-1425

6. Brown RD Jr, Evans BA, Wiebers DO, Petty GW, Meissner I, Dale AJD for the Mayo Clinic Division of Cerebrovascular Diseases: Transient Ischemic Attack and Minor Ischemic Stroke: An Algorithm for Evaluation and Treatment. Mayo Clin Proc 1994; 69:10271039

7. Benavente O, Moher D, Pham B: Carotid endarterectomy for asymptomatic carotid stenosis: a meta-analysis. BMJ, 1998; 317:1477-1480

8. Klijn CJM, Kappelle LJ, Tulleken CAF, van Gijn J: Symptomatic Carotid Artery Occlusion: a reappraisal of hemodynamic factors. Stroke 1997; 28(10):2084-2093.

9. Park AE, McCarthy WJ, Pearce WH, Matsumara JS, Yao JS: Carotid plaque morphology correlates with presenting symptomatology. J Vasc Surg 1998; 27 (5): 878-879

10. Nicolaides AN, Shifrin EG, Bradbury A, Dhanjil S, Griffin M, Belcaro G, Williams M: Angiographic and Duplex grading of internal carotid stenosis: can we overcome the confusion? J. Vasc. Endovasc Surg 1996; 3: 158 165.

11. Gent $\mathrm{M}$, Beaumont D, Blanchard $\mathrm{J}$ et al.: A randomised, blinded, trial of elopidogrel versus aspirin in patiens at risk of ischaemic events (CAPRIE). Lancet 1996; 348(9038): 1329-39.

12. Harbison JW: Ticlopidine versus aspirin for the prevention of recurrent stroke - analisys of patients with minor stroke from the ticlopidine aspirin stroke study. Stroke 1992; 23(12): 1723-1731.

13. Shahar E, Folsom AR, Romm FJ et al Patterns of aspirin use in middle-aged adults: the atherosclerosis risk in communities (ARIC) study. American Heart Journal 1996; 131(5): 915-922.

14. Nicolaides A: Asymptomatic Carotid Stenosis and Risk of Stroke (The ACSRS Study): Identification of a high risk group. proceedings of the 7th Meeting of the Mediterranean League of Angiology and Vascular Surgery. Limassol - Cyprus, 22-25/3/96, 1996, p.64-72. 